\title{
DISPOSITIVOS ANTIMIGRACIÓN DURANTE LA LITOTRICIA ENDOSCÓPICA CON LÁSER DE HOLMIUM:YAG
}

Pablo Garrido Abad, Inmaculada Fernández González, Almudena Coloma Del Peso, Milagros Jiménez Gálvez', Luis Miguel Herranz Fernández', Miguel Mora Durban, Gloria Bocardo Fajardo, Álvaro Serrano Pascual2, Lorenzo Herrero Torres e Ignacio Pereira Sanz.

Servicio de Urología del Hospital Universitario de La Princesa. Madrid.

'Servicio de Urología del Hospital del Henares. Coslada. Madrid.

2Servicio de Urología del Hospital Universitario de Guadalajara. Guadalajara. España.

Resumen.- OBJETIVO: La patología litiásica continúa siendo muy prevalente en nuestro medio. Existen múltiples enfoques y tratamientos para resolverla dentro de la urología actual.

En los últimos tiempos, las técnicas endourológicas han sufrido un espectacular avance que ha permitido aumentar su tasa de éxito, disminuyendo también de forma importante su comorbilidad. Sin embargo, a pesar de todas estas mejoras, nos encontramos aún con una serie de complicaciones que pueden reducir el éxito del procedimiento. Entre todas ellas, destacamos la retropulsión de la litiasis que ocurre durante la litofragmentación endoscópica, ya que aparece en un número importante de procedimientos $y$, no solo ocasiona un descenso del rango de "stone free", sino que además conlleva un alargamiento del tiempo quirúrgico y, en ocasiones, hará necesario el empleo de procedimientos adicionales que aumentarán el coste y la morbilidad del tratamiento.

Para reducir la incidencia de esta retropulsión se han empleado maniobras clásicas: antitrendelenburg, disminución de la intensidad de flujo de irrigación, modificación de los parámetros del láser o balones neumáticos. Actualmente disponemos de otros mecanismos más eficaces para combatirla, entre ellos: dispositivos que ocluyen la luz ureteral y cestillas/fórceps de nitinol.

La selección adecuada del dispositivo de extracción del cálculo puede ser decisiva para completar con éxito, y en el tiempo programado, un procedimiento ureteroscópico. La elección de un dispositivo inadecuado puede imposibilitar el procedimiento o causar un daño iatrogénico al tracto urinario.

Por todo ello, el instrumental específico para atrapar y extraer litiasis (cestillas y dispositivos que ocluyen la luz ureterall es un armamentarium con el que todo endourólogo debería estar familiarizado.

Palabras clave: Retropulsion. Litiasis ureteral. Láser.

Summary.- OBJECTIVES: Lithiasic pathology continues being very prevalent in our environment. There are multiple approaches and treatments to solve it in current urology.

Lately, endourological techniques have suffered a spectacular advance which has permitted to increase their success rates, diminishing also their comorbidity very much. Nevertheless, despite all improvements we still face a series of complications that may reduce the success of the procedure. Among all of them, we emphasize stone retropulsion during endoscopic lithotripsy, because it appears in an important number of procedures and, it does not only diminish the range of stone free 
patients, but also is associated with a longer surgical time, and occasionally will make necessary the employment of additional procedures that increase cost and treatment morbidity.

To reduce the incidence of this retropulsion classical manoeuvres have been employed: antitrendelenburg, decrease of the intensity of the irrigaton flow, modification of laser parameters, or pneumatic balloons. Currently, we have other more effective mechanisms to combat it, among them: the devices that occlude the ureteral lumen, and nitinol baskets/forceps.

Adequate selection of the stone extraction device may be definitive for completion of the ureterorenoscopic procedure with success, and in the programmed time. The election of an inadequate device may make the procedure impossible or cause iatrogenic damage to the urinary tract.

For all it, specific instruments to entrap and extract stones lbaskets and ureteral lumen occlusion devices) is an armamentarium that every endourologist should be familiar with.

Keywords: Retropulsion. Ureteral lithiasis. Laser.

\section{INTRODUCCIÓN}

En sus inicios la ureteroscopia se utilizó solamente como procedimiento diagnóstico, pero no transcurrió mucho tiempo antes de que se crearan instrumentos que la convirtieran en terapeútica también. Desde 1926 se han empleado cestas para atrapar y extraer cálculos ureterales. (1) En 1970 se desarrolló la cesta para cálculos de Dormia, y antes de los recientes avances en la tecnología de las cestas, era la más utilizada. (2) Antiguamente estas cestillas se fabricaban en acero inoxidable, que en la actualidad ha sido claramente sustituido por el nitinol. El nitinol es una aleación de niquel y titanio, que presenta la gran ventaja de su superelasticidad (alrededor de un 10\% más elástico que el resto de metales), y su capacidad de "recordar" su forma original tras una deformación completa. Todo ello, lo hace más útil para su manipulación ureteroscópica y atrapamiento/extracción de cálculos. (3) La mayoría de los dispositivos se encuentran recubiertos por una camisa de polyimide/PTFE. Con el tiempo, estos diámetros de las camisas, se han hecho lo suficientemente pequeños como para permitir su inserción a través de canales de trabajo de 3.6 Fr de los ureteroscopios flexibles, a la vez que han mantenido la calidad del dispositivo.

Recientemente, algunos investigadores han defendido la eliminación de la camisa que recubre las cestas de nitinol ("cestas desnudas") para disminuir su calibre y así mejorar, aún más, el rendimiento de los dispositivos (4). Sin embargo, a pesar de todos estos avances, nos encontramos todavía con una serie de complicaciones que pueden reducir el éxito del procedimiento. Entre todas ellas, destacamos la retropulsión de la litiasis que ocurre durante la litofragmentación endoscópica, ya que ocurre en un número importante de procedimientos $y$, no solo ocasiona un descenso del rango de "stone free", sino que además conlle- va un alargamiento del tiempo quirúrgico y, en ocasiones, hará necesario el empleo de procedimientos adicionales que aumentarán el coste y la morbilidad del tratamiento.

\section{TRATAMIENTO}

La litofragmentación mediante ureteroscopia se utiliza con éxito en urología desde hace varias décadas. Sin embargo no ha sido hasta los últimos tiempos, con la aparición de nuevo instrumental, cuando la tasa de éxito ha superado ampliamente a la mostrada por otras técnicas empleadas tradicionalmente, como la litotricia extracorpórea por ondas de choque (LEOC). La disponibilidad de ureteroscopios flexibles, guias de última generación y nuevas fuentes de fragmentación como el láser de Holmium:YAG (Ho:YAG) han facilitado la técnica, aumentando de manera muy importante el éxito del procedimiento y disminuyendo su tasa de complicaciones.

La retropulsión es el desplazamiento proximal de la litiasis debido a la acción de la energía de la fuente de fragmentación aplicada sobre la misma. La migración proximal de los cálculos durante la ureteroscopia continua siendo un problema frecuente, presente según algunos autores hasta en un $25 \%$ de los casos, incluso a pesar de la utilización de ureteroscopio flexible y láser Ho:YAG (5).

Otras diferentes fuentes de litofragmentación utilizadas durante la ureteroscopia como los ultrasonidos o la litotricia neumática presentan una tasa de migración proximal claramente superior a la mostrada por el láser de $\mathrm{Ho}$ : YAG. Marguet y cols. (6) en su estudio in vitro concluyen que tanto el Lithoclast como el láser Nd-YAG (FREDDY) provocan una retropulsión claramante superior a la del Ho: YAG. El mecanismo de fragmentación dominante en el láser Ho:YAG es fototermal, capaz de fragmentar todos los tipos de cálculos (independientemente de su composición), con menos efectos de tipo fotoacústico, lo que determina un menor desplazamiento de la litiasis durante el procedimiento $(7,8)$.

\section{MÉTODOS PARA DISMINUIR LA RETROPULSIÓN}

Para disminuir la frecuencia de esta complicación podemos aplicar diversos recursos, desde lo más sencillo hasta el instrumental más específico:

- Colocar al paciente en posición de antitrendelenburg.

- Disminuir la intensidad del flujo de irrigación.

- Podemos disminuir también la retropulsión con el láser de Ho:YAG modificando sus parámetros. Así a menor energía (julios) y frecuencia (hertzios) empleados produciremos menor retropulsión; del mismo modo que utilizando fibras de menor calibre (micras) y una amplitud de pulso (microsegundos) mayor (7).

- Dispositivos que ocluyen el uréter proximalmente para impedir la migración. El dispositivo Lithovac (MicrovasiveBoston Scientific Corp $®$ ) tiene una calibre demasiado gran- 
de para el uréter y el Lithocath (Microvasive-Boston Scientific Corp®.) y el Parachute (Microvasive-Boston Scientific Corp®.) son demasiado pequeños para uréteres dilatados, además de ocupar la mayor parte del canal de trabajo del ureteroscopio. Todos ellos con escasa capacidad para la recuperación de fragmentos tras la litotricia.

Por ello, en la actualidad se dispone de nuevos dispositivos más eficaces, como es el caso de los que presentamos a continuación:

\section{1) Dispositivos que ocluyen la luz ureteral:}

El Stone cone ${ }^{T M}$ de Boston Scientific Corporation ${ }^{\circledR}$ es un dispositivo antimigración compuesto de nitinol conformado por dos componentes: 1.- Espiral: diseñada para ocupar la luz ureteral y evitar así la migración proximal de litiasis durante el procedimiento. 2- Vaina: diseñada para resistir la energía láser Ho:YAG. Debido a su especial conformación permite el arrastre de múltiples restos litiásicos y su extracción en un sólo paso. Presenta una longitud de la vaina de $115 \mathrm{~cm}$., un calibre de $3.0 \mathrm{Fr}$, y dos posibles dimensiones de la espiral 7-10 mm. (Figuras 1A, 1B, 1C, 1D y $1 \mathrm{E})$.

El dispositivo $\mathbf{N}$-trap ${ }^{\circledR}$ de Cook Medica/尺 está compuesto también de nitinol y presenta una disposición distal en forma de paraguas que impide la migración litiásica. Presenta un calibre de $2.6 \mathrm{Fr}$. $(0.86 \mathrm{~mm}$.) y una longitud de $145 \mathrm{~cm}$. con un diámetro en el extremo distal de $7 \mathrm{~mm}$. (Figuras 2A, 2B y 2C).

\section{2) Cestillas y/o fórceps de nitinol:}

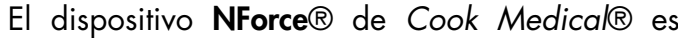
una cestilla de 3 radios. La superior consistencia de Delta
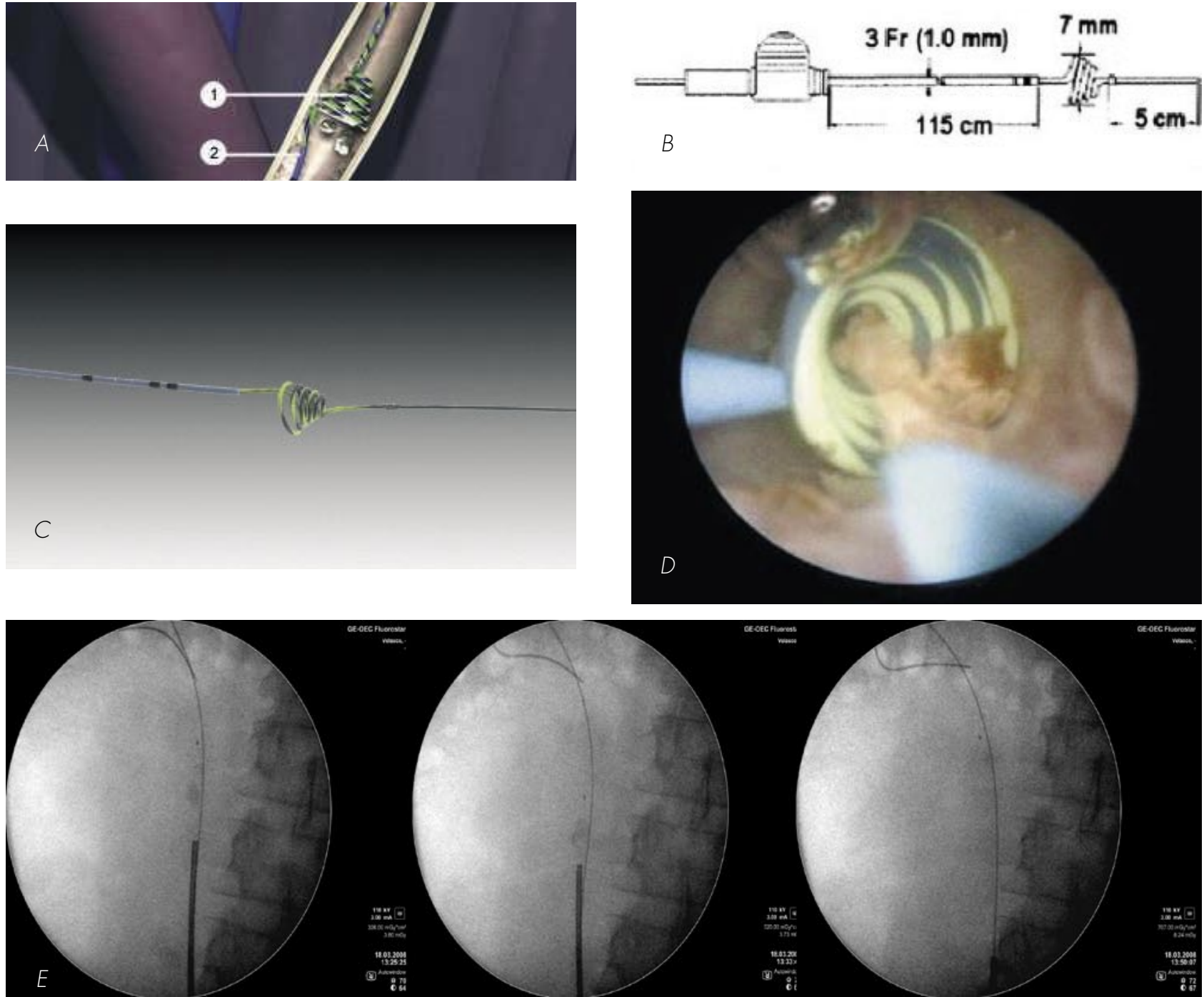

FIGURA 1A, IB y 1C. Diseño del Stone coneTM de Boston Scientific Corporation $\AA$

1D. Imagen endoscópica del Stone cone ${ }^{T M}$ de Boston Scientific Corporation $\AA$.

1E. Imagen en el amplificador de imágenes antes de la litotricia, durante y despues. 

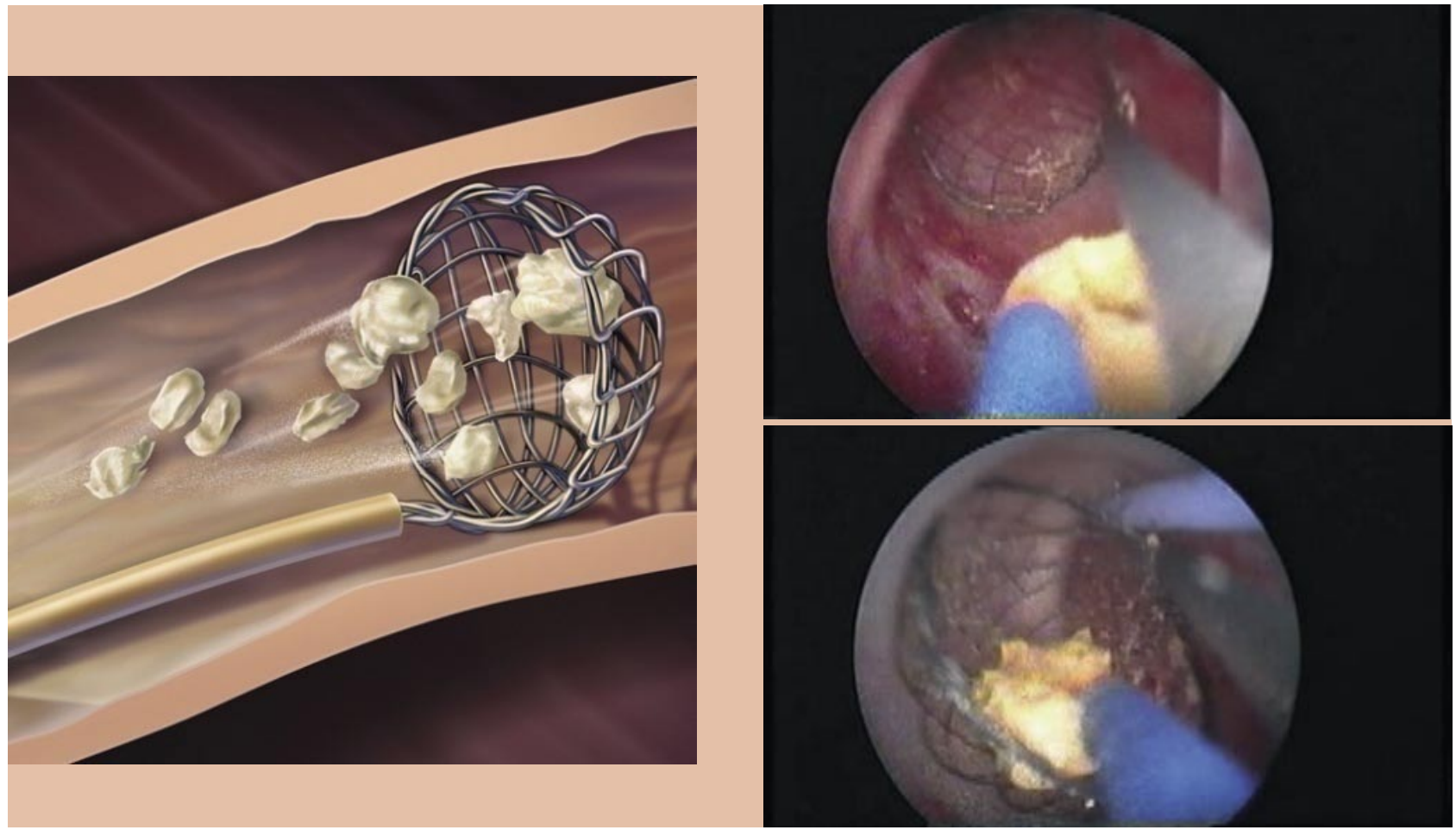

FIGURA 2A. Esquema del dispositivo N-trap $®$ de Cook Medical@

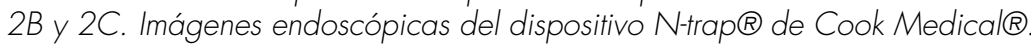

Wire $\AA$, combinada con la memoria del nitinol, crea una cestilla con una fuerza radial aumentada, lo que permite una mayor dilatación de la luz ureteral y atrapamiento de litiasis. Tiene una longitud de $115 \mathrm{cms}$, con cestillas de 5 ó $7 \mathrm{cms}$. Todo ello con 2 diferentes calibres disponibles. $(2,2$ y 3,2 Fr) (Figura 3).

El dispositivo NCompass $\AA$ de Cook Medical ( presenta la peculiaridad de una doble disposición. Cuando se despliega en su totalidad se comporta como una cestilla de 3 radios. Sin embargo, si se cierra parcialmente, se comporta como una cestilla de 12-16 radios. Esta dualidad permite la captura de litiasis durante la cirugía, disminuyendo así su movilidad, y la extracción de pequeños restos litiásicos tras la litotricia intracorpórea. Disponible en calibres de 1.7 y $2.4 \mathrm{Fr}$. Presenta una longitud de 115 cms. y diámetros de la cestilla disponibles en 1 y $1.5 \mathrm{cms}$. (Figura 4).

El dispositivo NCircle ${ }^{\mathrm{TM}}$ de Cook Medical $\AA^{\circledR}$ aporta la memoria de la guía de nitinol, que permite a la cestilla mantener su forma tras la torsión extrema. El diseño romo del modelo tipless ayuda a la captura de litiasis caliciales permitiendo a la cestilla abrirse en la base del cáliz y atrapar el cálculo. Además, disminuye el riesgo de daño o perforación de la mucosa ureteral. Las versiones en 1.5 y 2.2 Fr presentan un calibre y una flexibilidad que permiten una deflexión completa y prolongada del ureteroscopio flexible con menor disminución del flujo de irrigación durante la manipulación de la litiasis. Disponible también el modelo helical tipless con calibres de 3.0, 3.2 y 4.5 Fr. Presenta longitudes de 65 y $115 \mathrm{cms}$. y diámetros de la cestilla en 1.0 y $2.0 \mathrm{cms}$. (Figuras 5A y 5B).

El dispositivo Escape ${ }^{\circledR}$ de Boston Scientific (Microvasive) $(\mathbb{R}$ permite una manipulación segura e independiente de la cestilla de nitinol y de la fibra láser de holmio. El calibre de $1.9 \mathrm{Fr}$ permite un flujo de irrigación superior a los de 2.2 y $2.4 \mathrm{Fr}$. Cuando se introducen a través de un canal de trabajo de $3.6 \mathrm{Fr}$, la guía de $1.9 \mathrm{Fr}$ y la fibra láser

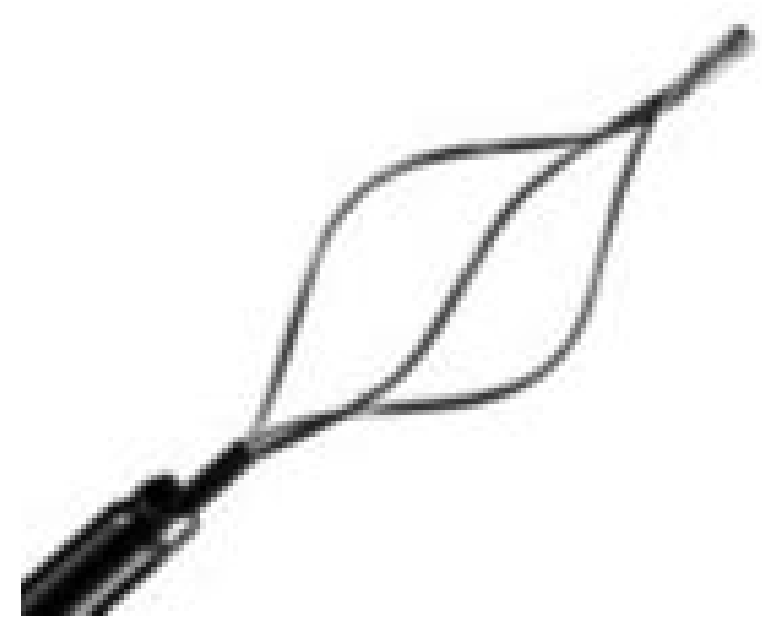

FIGURA 3. Diseño del dispositivo NForce® de Cook Medical巴 


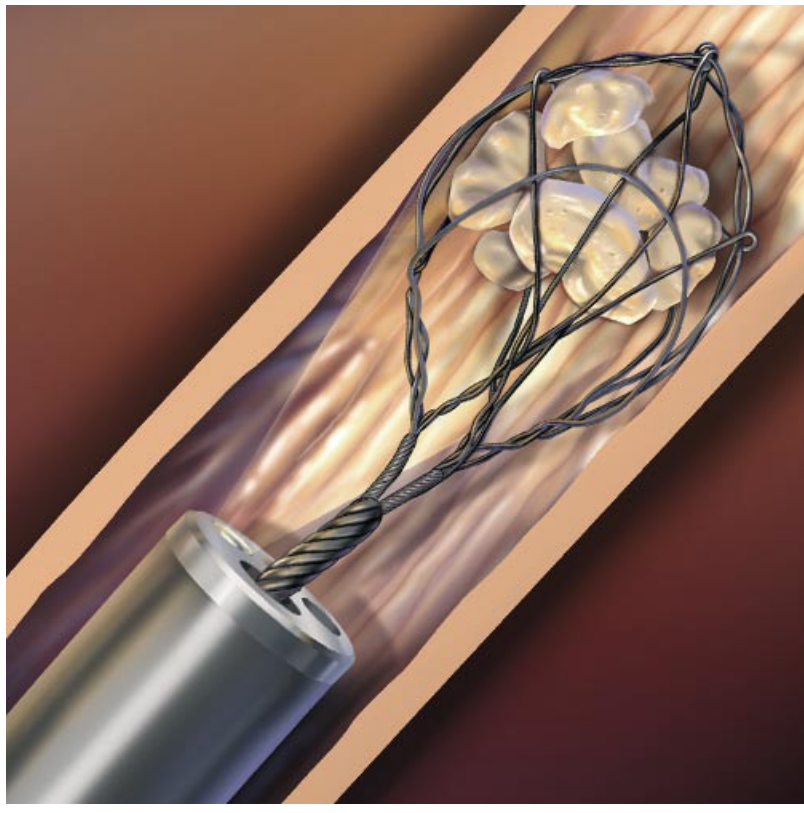

FIGURA 4. Diseño del dispositivo NCompass® de Cook Medical尺.

de $200 \mu \mathrm{m}$ se consigue un flujo de irrigación equivalente a un dispositivo de $2.4 \mathrm{Fr}$. El diseño de la cestilla de nitinol roma integra 2 conformaciones. En su posición habitual presenta un radio de $11 \mathrm{~mm}$. Sin embargo, en la posición de máxima apertura, se extienden además 2 patas accesorias que facilitan la liberación (15 mm). (Figuras 6A y 6B).
El dispositivo ZeroTip ${ }^{\mathrm{TM}}$ de Boston Scientific (Mi-

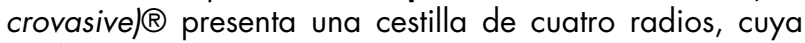
configuración permite la cómoda extracción de litiasis ureterales. Su superficie lisa distal permite además una manipulación atraumática y sin fricciones. Su composición combinada de polyimide/PTFE (vaina) y nitinol (cestilla) aporta resistencia y flexibilidad para maximizar la posible deflexión del ureteroscopio. Su longitud varía de 90 a 120 cms en función del modelo (Figuras 7A, 7B y 7C).

El dispositivo Graspit $₫$ de Boston Scientific (Microvasive) $(\mathbb{R}$ es, en realidad, más un fórceps que una cestilla. La configuración del fórceps (disponible en 8 y 10 $\mathrm{mm}$.), con superficie dentada, produce un agarre eficaz y un manejo seguro de la litiasis. La composición en nitinol del mismo, aporta resistencia y flexibilidad, permitiendo la deflexión y su manipulación intracalicial. Su vaina de baja fricción permite un sencillo manejo por el canal de trabajo del instrumental y evita lesiones ureterales. Su longitud varía entre 90 y $120 \mathrm{cms}$, en función del modelo elegido. (Figuras $8 \mathrm{~A}, 8 \mathrm{~B}$ y $8 \mathrm{C}$ ).

El dispositivo Sur-Catch ${ }^{\text {TM }}$ NT de Gyrus ACM/® presenta también las ventajas de una cestilla de nitinol, de tipo tipless, con la peculiaridad de su diseño dual $(3 x$ 2 radios, y $16 \mathrm{~mm}$ de diámetrol y de capacidad variable lo que permite ajustarse de manera más precisa a cada tamaño de litiasis. Su longitud es de $120 \mathrm{~cm}$. y su calibre de $2.2 \mathrm{Fr}$. Aporta un mango ergonómico que facilita su manejo. Está diseñado para ser usado específicamente con el ureteroscopio DURTM-8 Elite. Por otra parte, la cestilla SurLok ${ }^{\text {TM }}$ plana/helicoidal de Gyrus ACMI尺 tiene un diseño estándar/espiral, con $4 / 6$ radios y $8.5,10,11,16,20$ y $30 \mathrm{~mm}$ de diámetro, que facilita la captura de la litiasis

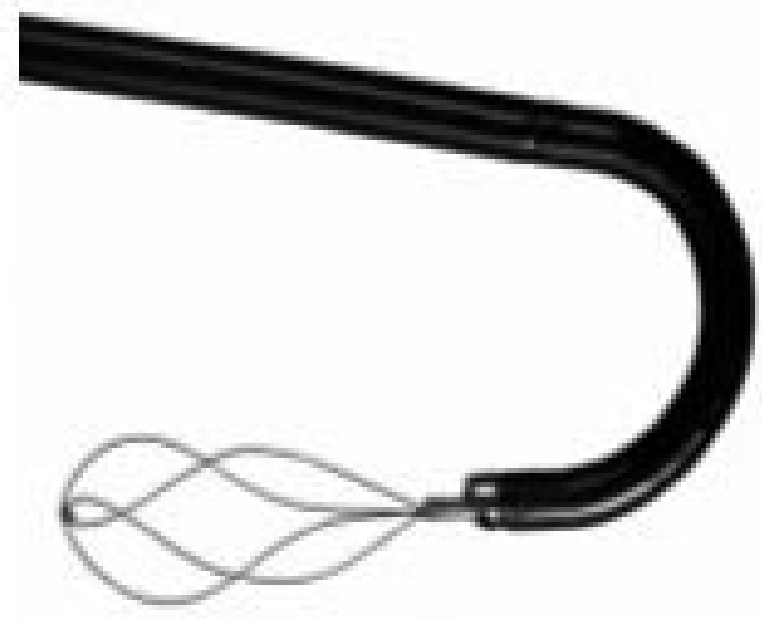

A

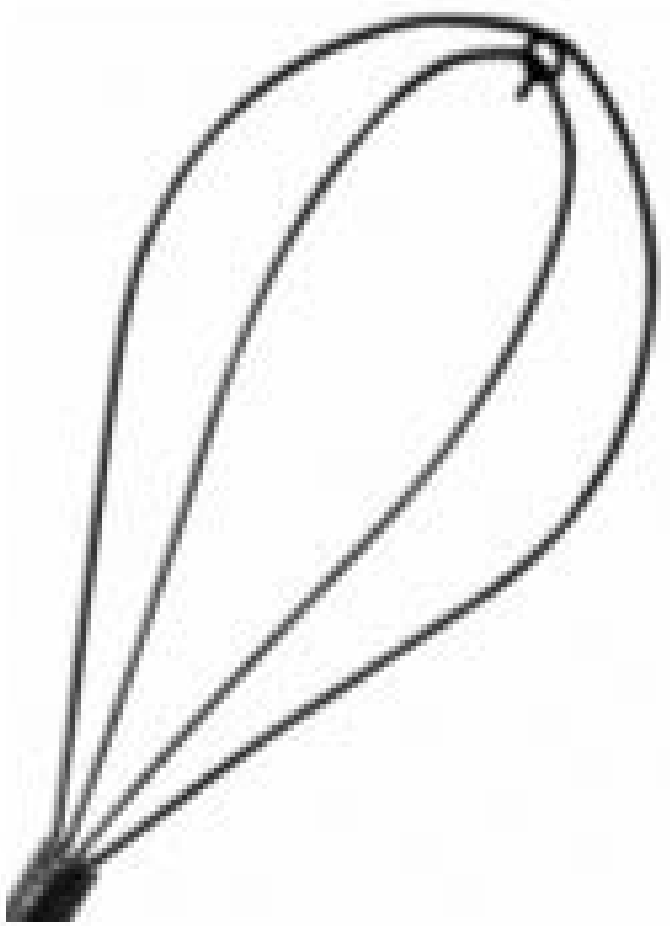



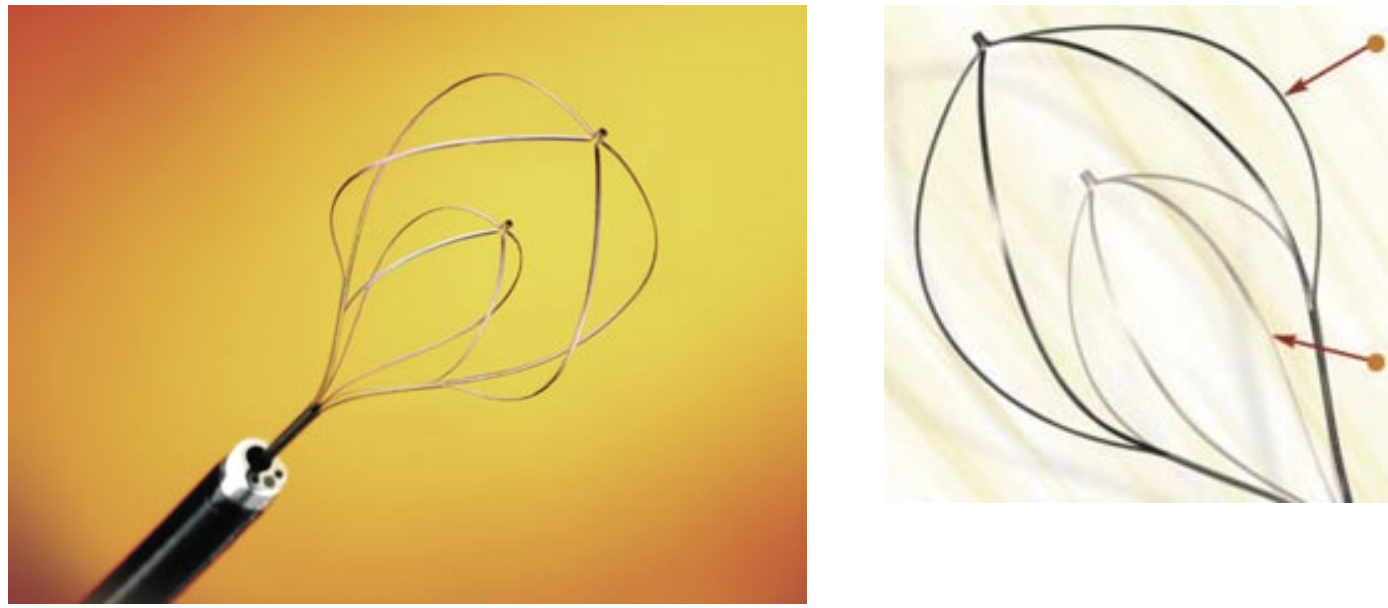

FIGURA 6A y 6B. Diseño del dispositivo Escape ${ }^{\circledR}$ de Boston Scientific (Microvasive) $尺$.

y su extracción con eficacia y seguridad. Sus diferentes modelos presentan longitudes de 70,90 y $120 \mathrm{cms}$, con calibres de $2.4,3.0$ y 4.5 Fr. (Figuras 9A y 9B).

La cestilla de nitinol (B9002) de Applied Medical® presenta un calibre de $3.0 \mathrm{Fr}$, una longitud de 120 cms y un diámetro de 12-14 mm. Permiten una extracción segura y eficaz de las litiasis alojadas en la luz ureteral con sencilla manipulación del dispositivo (Figura 10).
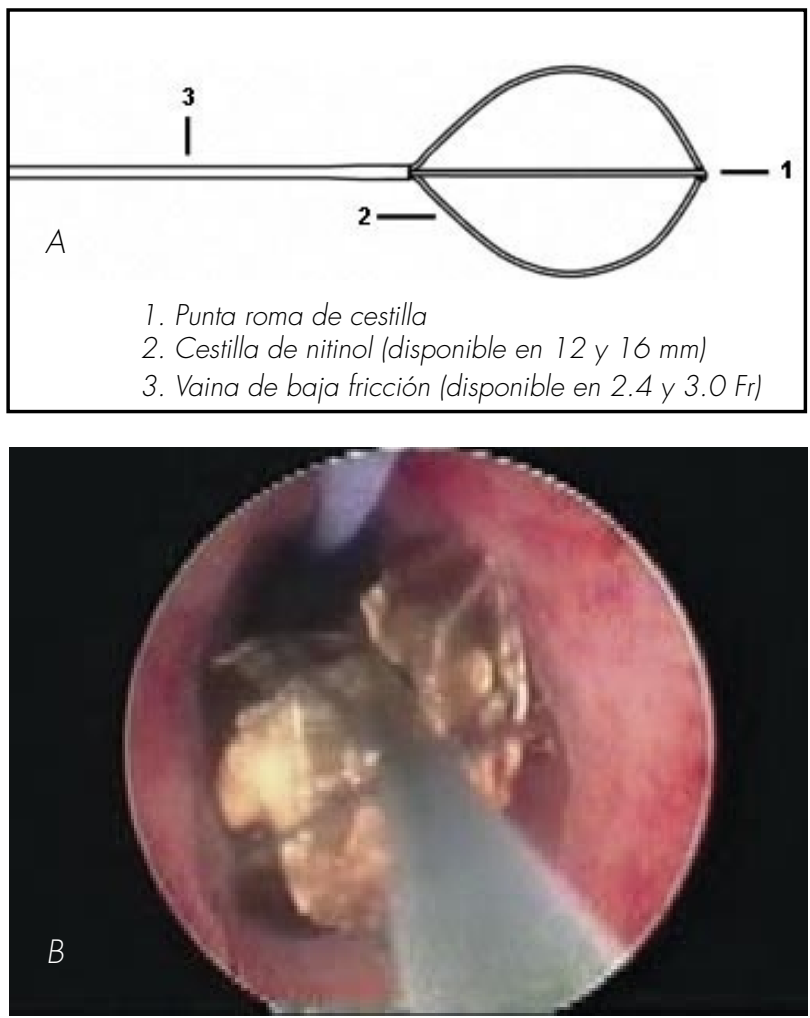

El dispositivo Bard $\circledast$ Dimension ${ }^{\mathrm{TM}}$ de Bard Urologicalß está compuesto de nitinol y presenta la peculiaridad de ser articulado, permitiendo su uso como cestilla o grasper en un mismo instrumento. Mejora así la captura y extracción de litiasis en difíciles localizaciones. Su longitud es de $115 \mathrm{cms}$, su calibre de 2.4 y $3.0 \mathrm{Fr}$, con diámetros de cestilla de 10, 13 y $16 \mathrm{~mm}$. (Figuras 11 a y $11 \mathrm{~b}$ ). La cestilla Expand212 $\circledast$ compuesta de nitinol, es de tipo helicoidal (con y sin punta), con calibre de $3.0 \mathrm{Fr}$, longitud de 90-115 cms y un diámetro de $11 \mathrm{~mm}$. (Figura 11c).

Bard Urological $($ dispone además de (1): Cestillas de nitinol denominadas Platinum Class ${ }^{\mathrm{TM}}$ a) Torque-Stable de tipo helicoidal con una longitud de 70, 90 y 115 cms, calibre de $3.0 \mathrm{Fr}$ y diámetro de $14 \mathrm{~mm}$. Y b) Flat wire con una longitud de 70, 90 y $115 \mathrm{cms}$, calibres de 2.4 y $3.0 \mathrm{Fr}$ y diámetro de 14-18 mm. (2): Fórceps Platinum Class $^{\mathrm{TM}}$ Non-Retracting Grasping con unas longitudes de 90 y $115 \mathrm{cms}$ y calibre de 3.0 Fr. (Figuras $12 \mathrm{a}$ y $12 \mathrm{~b}$ ).

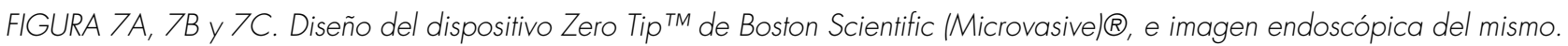



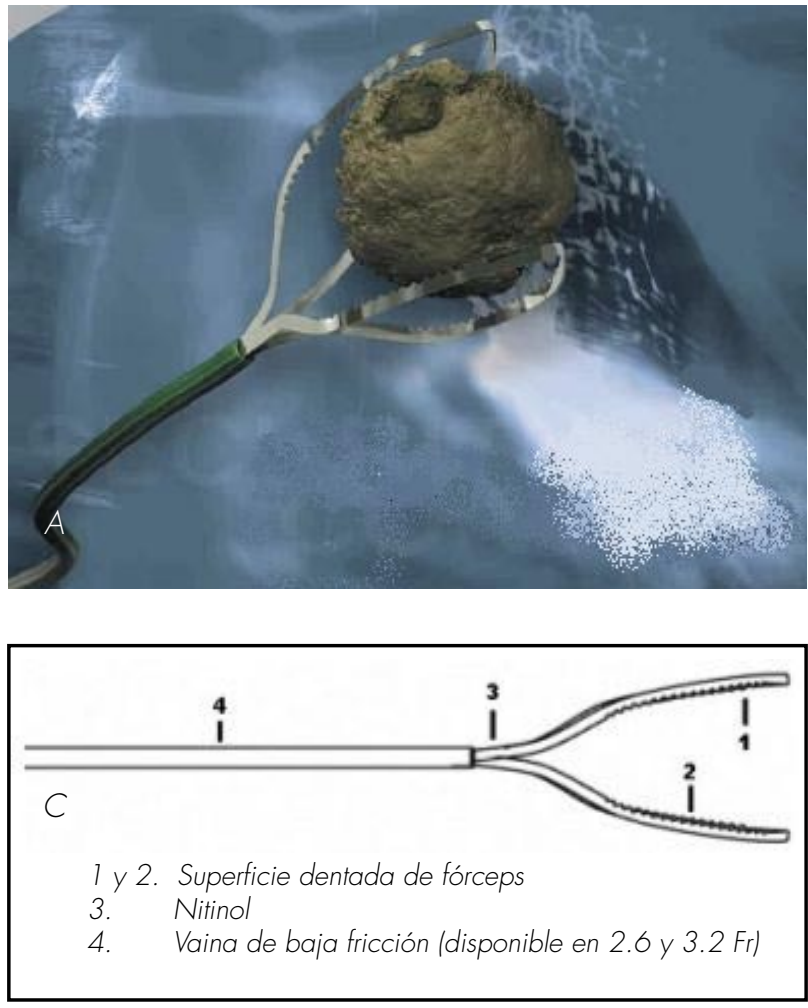

FIGURA 8A, 8B y 8C. Diseño del dispositivo Graspitß de Boston Scientific (Microvasive) $($.

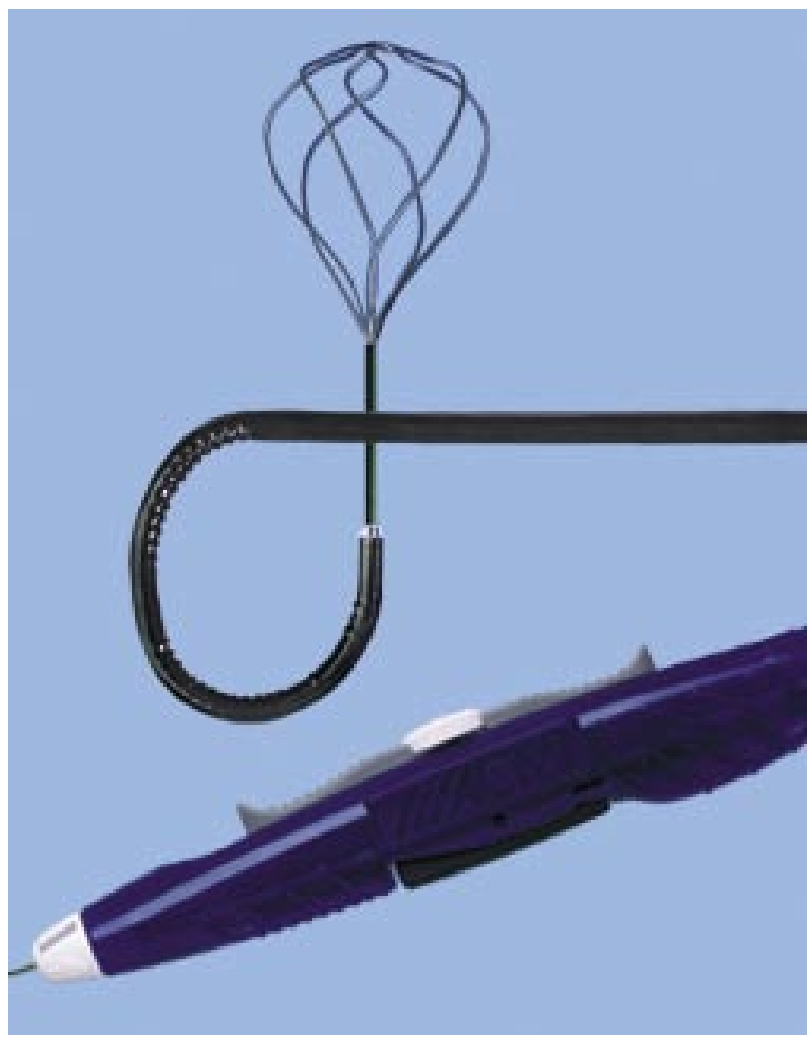

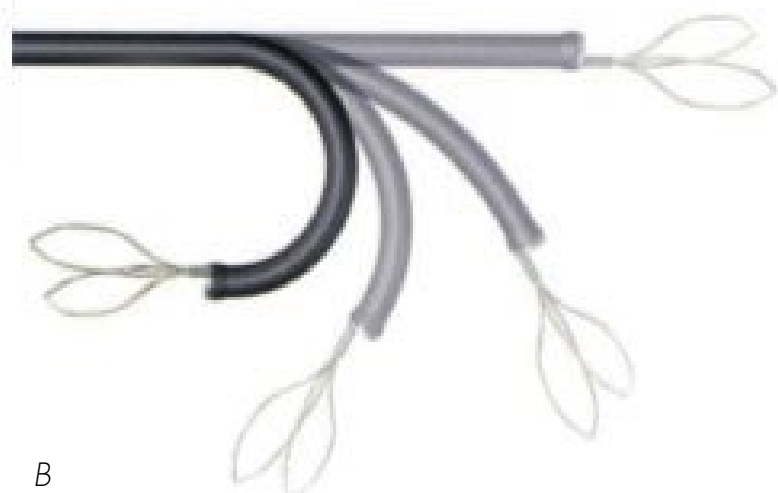

La cestilla de nitinol HALOTM de Sacred Heart Medical@ presenta una longitud de $90-120 \mathrm{cms}$, un diámetro de cestilla de $12 \mathrm{~mm}$ y un calibre de $1.5 \mathrm{Fr}$. Su escaso calibre le permite mejorar mucho el flujo de irrigación a través del ureteroscopio y facilitar así el manejo de la litiasis ureteral (Figura 13A y 13B).

Sacred Heart Medical@ dispone también de otras cestillas: 1) Vantage ${ }^{\mathrm{TM}}$, con una longitud de 90-120 cms, un calibre de 2.4-3.0 Fr y un diámetro de cestilla de 16 mm. 2) Hercules $^{\mathrm{TM}}$, helicoidal doble, de acero inoxidable,

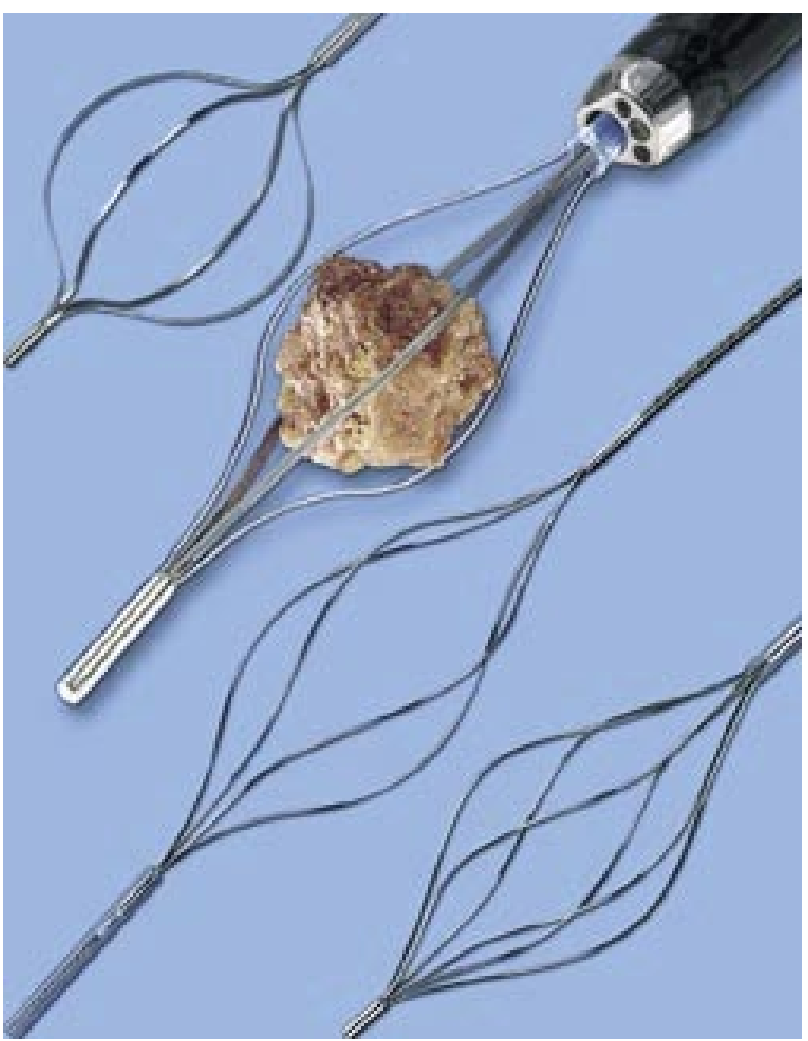

FIGURAS 9A y 9B. Diseño de cestillas Sur-catch ${ }^{T M}$ y Surlok ${ }^{T M}$, respectivamente, de Gyrus ACMI@. 


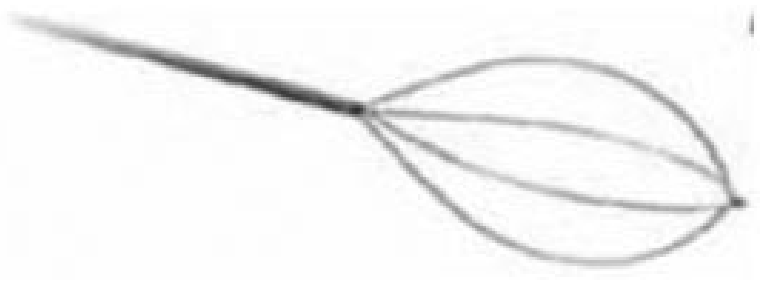

FIGURA 10. Diseño de la cestilla de nitinol B9002 de Applied Medical@.

con un calibre de $3.0 \mathrm{Fr}$, longitud de $90-120 \mathrm{cms}$ y un diámetro de la cestilla de $11 \mathrm{~mm}$. (Figuras 14A y 14B).

El dispositivo NGage ${ }^{\mathrm{TM}}$ de Cook Medical ${ }^{\circledR}$ es un instrumento desechable, fabricado en nitinol, que tiene un diámetro de tan sólo 1.7/2,2 $\mathrm{Fr}$ y una longitud de 115 $\mathrm{cms}$. Consta de tres ramas que se abren y cierran a modo de tridente, con la particularidad de que sus extremos distales están unidos entre sí mediante otros finos hilos de nitinol. Esto hace que tenga una forma de cono abierto (disponible en 8 y $11 \mathrm{cms}$ ) hacia el cálculo, con la capacidad de iniciar su cierre precisamente por su base (Figura 15).
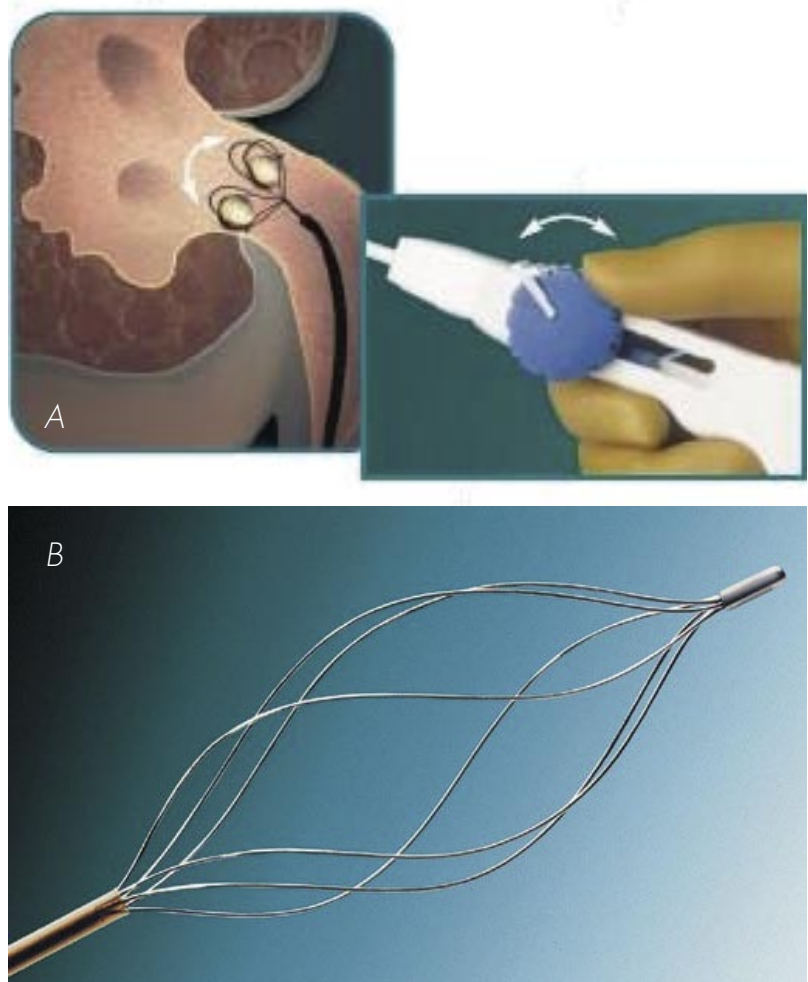

\section{DISCUSIÓN}

La patología litiásica es una de las más prevalentes en nuestro medio. Su enfoque terapeútico se ha modificado radicalmente en los últimos tiempos con el imparable desarrollo de la endourología. En los últimos años el avance en el diseño y fabricación de instrumental endourológico ha evolucionado de forma muy importante. Los ureteroscopios son cada vez más pequeños, flexibles y precisos para resolver la patología litiásica con la mínima iatrogenia durante el procedimiento.

Sin embargo uno de los principales problemas durante la litotricia endoscópica es la retropulsión que se produce al impactar una fuente de energía sobre la litiasis. Supone un aumento del tiempo quirúrgico y de la posible iatrogenia. Todo ello puede llevar a un fracaso de la técnica.

Este problema se ha minimizado en la actualidad con la aparición de distintos dispositivos que, introducidos a través del ureteroscopio, evitan la migración litiásica proximal durante la litotricia endoscópica. Pero el instrumental que se introduce a través del canal de trabajo debe continuar permitiendo una fácil maniobrabilidad, con la menor disminución de flujo de irrigación. Esto se ha conseguido con:

1) La aparición de nuevos materiales como el nitinol, que aporta resistencia, flexibilidad y memoria, sin disminuir de forma importante la capacidad de deflexión del instrumental de tipo flexible.

2) La disminución del calibre, que permite un mayor flujo de líquido de irrigación, con la consiguiente mejora en la visibilidad durante el procedimiento.

En la actualidad disponemos de tanta variedad de instrumental que es difícil seleccionar, de entre todos ellos, aquellos con los que obtendremos mejores resultados. El caso de los dispositivos diseñados para evitar la migración litiásica durante la ureteroscopia no representa una excepción. Existe un gran número y diversidad de instru-

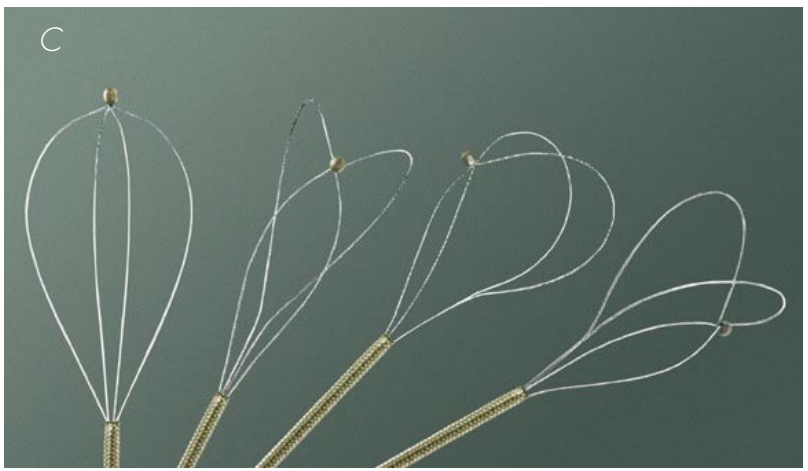

FIGURAS 1 1A y 1 1B. Diseño y manejo del dispositivo Bard@ Dimension ${ }^{T M}$ de Bard Urological $\Theta$. 11C. Diseño cestilla Expand $12 \AA$ de Bard Urological $($. 

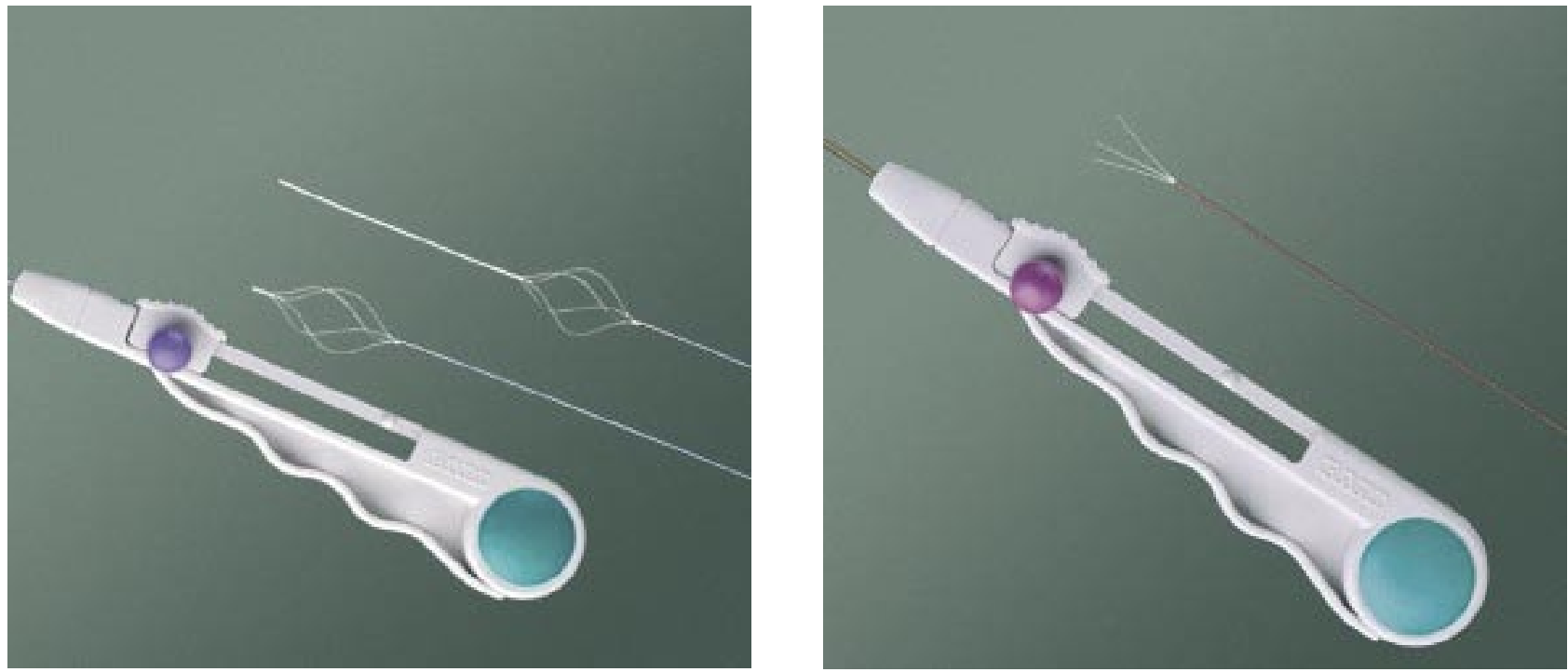

FIGURAS 12A y 12B. Dispositivos Platinum class ${ }^{T M}$ (cestilla y forceps) de Bard Urologicale.
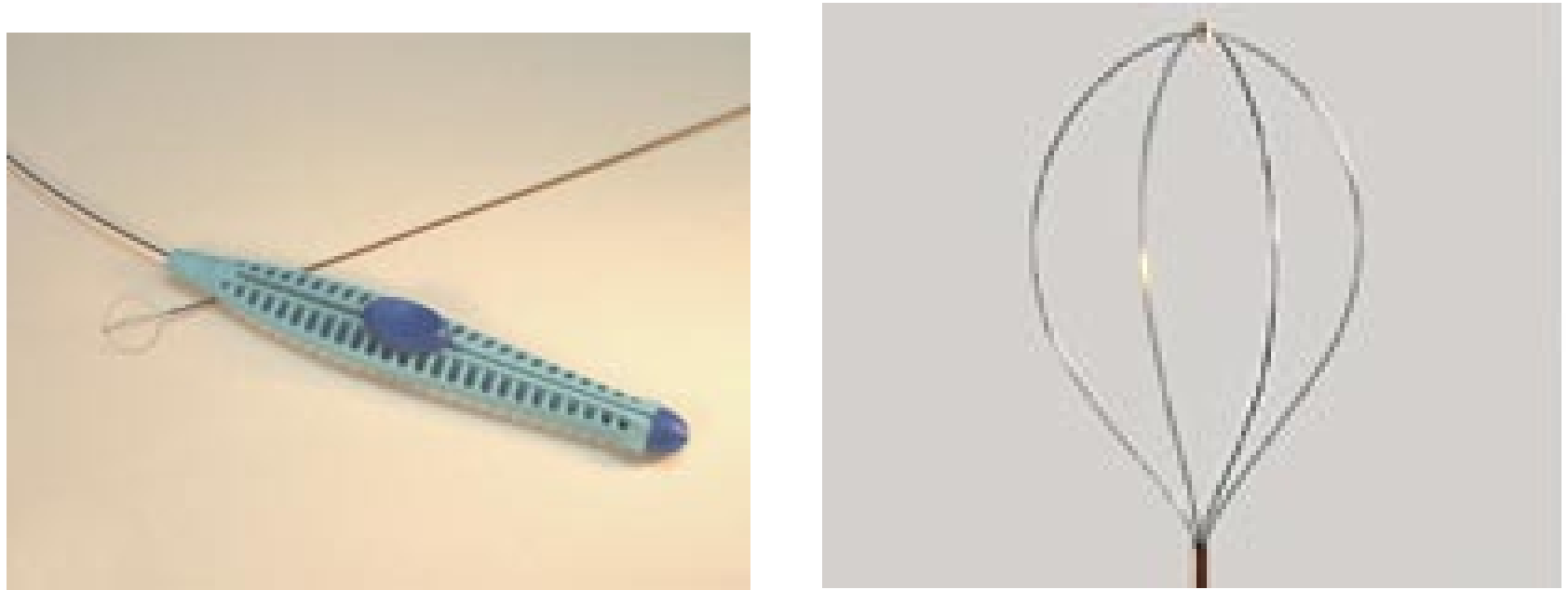

FIGURAS 13A y 13B. Diseño de la cestilla de nitinol HALOTM de Sacred Heart Medicale
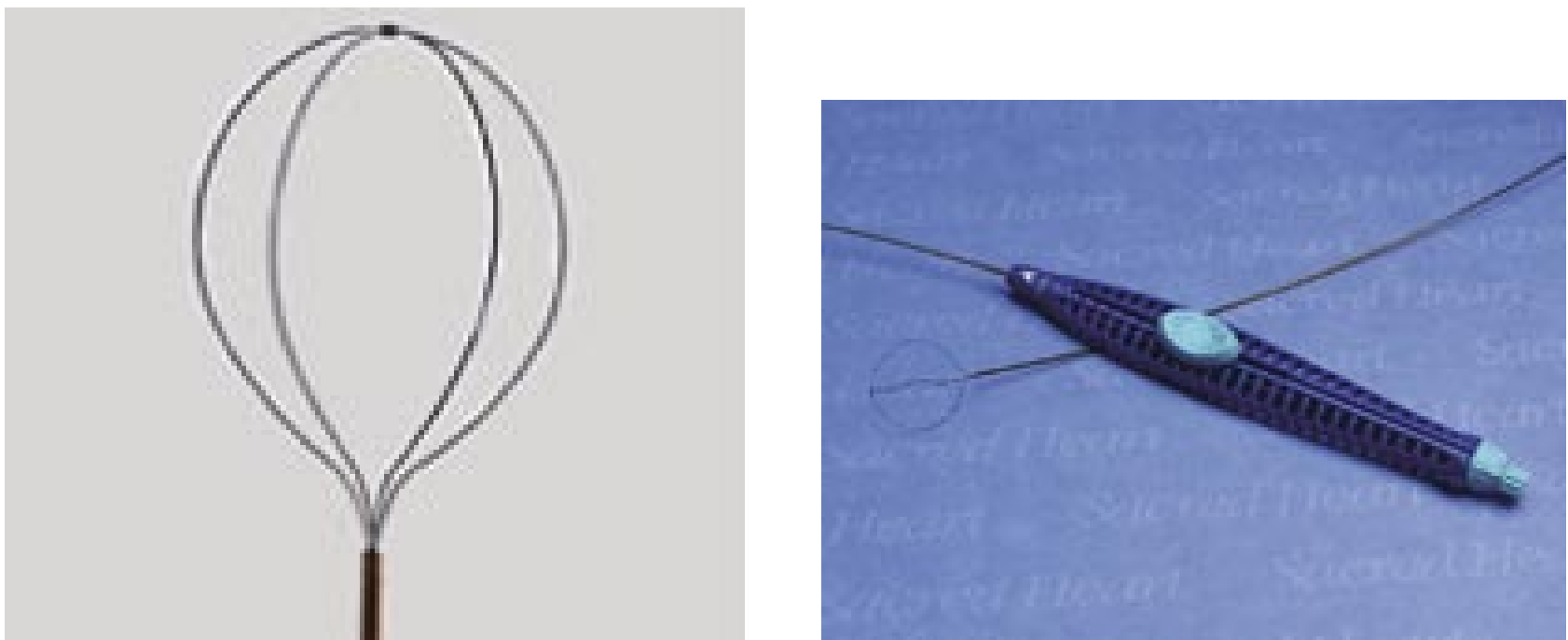

FIGURAS 14A y 14B. Diseño de las cestillas Vantage ${ }^{T M}$ y Hercules ${ }^{T M}$ de Sacred Heart Medical ${ }^{\circledR}$. 
mental aplicado a este fin (incluyendo sistemas ocluyentes de la luz ureteral, cestillas y fórceps/graspers). Debemos valorar no sólo sus características técnicas, sino también otros parámetros como manejabilidad, biocompatibilidad, disponibilidad y precio.

En la literatura actual no es fácil encontrar estudios fiables donde se empleen análisis estadísticos que comparen todos estos distintos dispositivos.

En lo referente al instrumental ocluyente de la luz ureteral debemos destacar el trabajo de Rodríguez y cols. (9) donde concluyen que este tipo de dispositivos suponen un descenso en la necesidad de practicar repetidas manipulaciones ureterales, así como un método más sencillo y seguro para la extracción de fragmentos litiásicos. El Stone Cone $^{\mathrm{TM}}$ parece tener más ventajas sobre las cestillas tipo Dormia durante la litotricia ureteroscópica, en términos de menor incidencia de fragmentos residuales y de necesidad de reintervenciones o técnicas auxiliares. Por su parte Holley y cols. (10) y Finley y cols. (11) comparan el Stone Cone ${ }^{T M}$ y el N-Trap ${ }^{T M}$, concluyendo que ambos son dispositivos muy eficaces evitando la migración litiásica retrógrada, siendo capaces de retener todos los fragmentos litiásicos $>2.5 \mathrm{~mm}$. Sin embargo el N-Trap ${ }^{T M}$ es el único capaz incluso de retener fragmentos de entre 1 y $2.5 \mathrm{~mm}$, (especialmente en su zona central).

En cuanto a las cestillas de nitinol, Money y cols. (12) demuestra que las cestillas del tipo tipless son superiores, con mejores resultados, menores tiempos operatorios y frecuencia de lesiones iatrogénicas frente a otros diseños de cestillas.

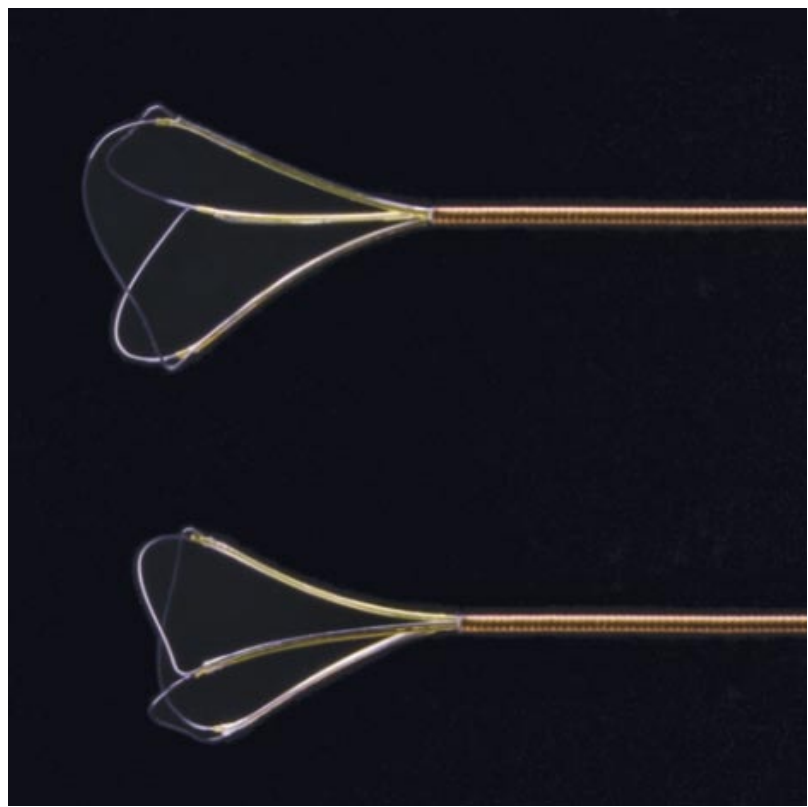

FIGURA 15. Diseño del dispositivo NGage ${ }^{T M}$ de Cook Medical $R_{\text {. }}$
Encontramos otros trabajos interesantes como el de Monga y cols. (13), El-Gabry y cols. (14) y Lukasewycz y cols. (15) donde se comparan in vitro las cestillas de nitinol de tipo tipless (sin punta) más frecuentemente utilizadas. Comparan el tiempo empleado para la extracción litiásica con todas ellas en función del tamaño del cálculo. Se comparan entre sí varias cestillas: Sur-Catch ${ }^{\mathrm{TM}} 3.0 \mathrm{Fr}$, ZeroTip $^{\mathrm{TM}}$ 3.0-2.4 Fr, N-Circle ${ }^{\mathrm{TM}}$ 3.3-3.0-2.2 Fr, Dimen$\operatorname{sion}^{\mathrm{TM}} 3.0 \mathrm{Fr}, \mathrm{HALO} \mathrm{TM}^{\mathrm{TM}} 1.9 \mathrm{Fr}$ y Vantage ${ }^{\mathrm{TM}} 1.9 \mathrm{Fr}$.

Concluyen que, para las litiasis de menor tamaño (2 mm.) el Sur-Catch ${ }^{\mathrm{TM}}$ es claramente el más lento, pudiendo estar relacionado con su conformación helicoidal de 6 radios, que dificulta el atrapamiento. Para las litiasis de mediano tamaño $\left(2-5 \mathrm{~mm}\right.$.), el HALO ${ }^{\mathrm{TM}}$ y Vantage $\mathrm{V}^{\mathrm{TM}}$ tienen las mejores características para su extracción. Parece puede estar relacionado con la característica de su nitinol trenzado que permite una mayor deformabilidad y atrapamiento del cálculo. En el caso de las litiasis de mayor tamaño (8 mm.) el ZeroTip ${ }^{\mathrm{TM}}$ y el Dimension ${ }^{\mathrm{TM}}$ obtienen los mejores resultados, mientras que el Sur-Catch ${ }^{\mathrm{TM}}, \mathrm{HALO}^{\mathrm{TM}}$ y Vantage $^{\mathrm{TM}}$ son los más lentos.

Si comparamos el impacto sobre la irrigación y deflexión a través del ureteroscopio, es importante conocer que una irrigación a presión suficiente para proprocionar una visión adecuada es fundamental para el éxito del procedimiento. (16) Por ello debemos reseñar los artículos de Nagele y cols. (17) y Ames y cols. (18) donde se comparan diferentes dispositivos.

En el primero, Nagele y cols, utilizando un ureteroscopio flexible de última generación concluye que los dispositivos de menor calibre producen una disminución significativamente menor del flujo de líquido de irrigación. Así, el HALO ${ }^{\mathrm{TM}} 1.5 \mathrm{Fr}$ permite alrededor de un $40 \%$ del flujo total, incluso en situación de deflexión 180․ Mientras el ZeroTip ${ }^{\mathrm{TM}}$ de $1.9 \mathrm{Fr}$ permite un $30 \%$ del flujo, el N-Circle $^{\mathrm{TM}}$ de $2.2 \mathrm{Fr}$ un $25 \%$, el ZeroTip ${ }^{\mathrm{TM}}$ de $2.4 \mathrm{Fr}$ un $7 \%$ y el Vantage $^{\mathrm{TM}}$ de $3 \mathrm{Fr}$. tan sólo un $1.2 \%$.

En el segundo de ellos, Ames y cols. describen que el flujo de irrigación a través del ureteroscopio y su capacidad de deflexión disminuyen progresivamente a medida que progresa el calibre de las cestillas, para concluir que la cestilla Microvasive $® 1.9 \mathrm{Fr}$ y la de Cook $(2.2$ Fr son las que menor disminución del flujo de irrigación y deflexión presentan. Bhayaniy cols. (19) refieren por su parte que, empleando cestillas "desnudas" (sin camisa), se podría aumentar la deflexión hasta en $15^{\circ}-20^{\circ}$ y del 2 al $30 \%$ del flujo de irrigación.

Seguramente serán necesarios nuevos trabajos que comparen dispositivos de igual o similar calibre puestos en el mercado por los distintos proveedores para poder evaluarlos de forma más significativa y precisa.

\section{CONCLUSIÓN}

La selección adecuada del dispositivo de extracción del cálculo puede ser decisiva para completar con éxito y en el tiempo programado un procedimiento urete- 
roscópico. La elección de un dispositivo inadecuado puede imposibilitar el procedimiento o causar un daño iatrogénico al tracto urinario (20).

Por todo ello, el instrumental específico para atrapar y extraer litiasis (cestillas y dispositivos que ocluyen la luz ureteral) es un armamentarium con el que todo endourólogo debería estar familiarizado.

\section{BIBLIOGRAFÍA y LECTURAS RECOMENDADAS ("lectura de interés $\mathrm{y}^{* *}$ lectura fundamental)}

1. COUNCILL, W.A.: "A new ureteral stone extractor and dilator”. JAMA, 86: 1907, 1926.

2. DORMIA, E.: "Dormia basket: Standard technique, observation and general concepts". Urology, 20: 437, 1982.

3. GOTMAN, I.: "Characteristic of metals used in implants". J. Endourol., 11: 383, 1997.

*4. LANDMAN, J.; MONGA, M.; EL-GABRY, E.A. y cols.: "Bare naked baskets: Ureteroscope deflection and flow characteristics with intact and disassembled ureteroscopic nitinol stone baskets". J. Urol., 56: 935, 2002.

*5. CHOW, G.; BLUTE, M.L.; PATTERSON, D.E. y cols.: "Ureteroscopy: Update on current practice and long term complications". J. Urol., 165: 71, 2001.

**6. MARGUET, C.G.; SUNG, J.C.; SPRINGHART, W.P. y cols.: "In vitro comparison of stone retropulsión and fragmentation of the frequency doubled, doublé pulse Nd: YAG laser and the Holmium: YAG laser". J. Urol., 173: 1797, 2005.

7. CHAN, K.F.; VASSAR, G.J.; PFEFER, T.J. y cols.: "Holmium: YAG laser lithotripsy: A dominant phototermal ablative mechanism with chemical decomposition of urinary calculi”. Laser Surg. Med., 25: 22, 1999.

8. YIU, M.K.; LIU, P.L.; YIU, T.F. y cols.: "Clinical experience with holmium: YAG laser lithotripsy of ureteral calculi". Laser Surg. Med., 19: 103, 1996.
9. RODRÍGUEZ GARCIA, N.; FERNÁNDEZ GONZÁLEZ, I.; PASCUAL MATEO, C. y cols.: "Stone Cone $^{\mathrm{TM}}$ : Un dispositivo que previene la migración de litiasis ureteral durante la litotricia intracorpórea". Arch. Esp. Urol., 58: 329, 2005.

**10. HOLLEY, P.G.; SHARMA, S.K.; PERRY, K.P. y cols.: "Assessment of novel ureteral oclusión device and comparison with stone cone in prevention of stone fragment migration during lithotripsy". J. Enourol., 19: 200, 2005.

*11. FINLEY, D.S.; PETERSEN, J.; ABDELSHEHID, C. y cols.: "Effect of holmium: YAG laser pulse width on lithotripsy retropulsion in vitro". J. Endourol., 19: 1041, 2005.

12. HONEY, J.R.: "Assessment of a new tipless nitinol stone basket and comparison with an existing flat-wire basket". J. Endourol., 12: 529, 1998.

13. MONGA, M.; HENDLIN, K.; LEE, C. y cols.: "Systematic evaluation of stone basket dimensions". Urology, 63: 1042, 2004.

14. EL-GABRY, E.A.; BAGLEY, D.H.: "Retrieval capabilities of different stone basket designs in vitro". J. Endourol., 13: 305, 1999.

*15. LUKASEWYCZ, S.; HOFFMAN, N.; BOTNARU, A. y cols.: "Comparison of tipless and helical baskets in an in vitro ureteral model". Urology, 64: 435, 2004.

16. PARKIN, J.; KEELY, F.X.; TIMONEY, A.G.: "Flexible ureteroscopes: A user's guide”. Br. J. Urol., 90: 640, 2002.

17. NAGELE, U.; PRAETORIUS, M.; SCHILLING, D. y cols.: "Comparison of flexible grasping forceps and stone basket for removal of retracted ureteral stents". J. Endourol., 20: 418, 2006.

*18. AMES, C.D.; PERRONE, J.M.; WELD, K.J. y cols. "Alteration in irrigant flow and deflection of flexible ureteroscopes with nitinol baskets". J. Endourol., 20: 74, 2006

19. BHAYANI, S.B.; MONGA, M.; LANDMAN, J. y cols.: "Bare naked baskets: Optimizing ureteroscopic stone extraction". Urology, 60: 147, 2002.

**20. ROSENBERG, B.H.; AVERCH, T.D.: "Ancillary instrumentation for ureteroscopy". Urol. Clin. North Am., 31: 49, 2004. 